Research

\title{
$B$ cells and monocytes from patients with active multiple sclerosis exhibit increased surface expression of both HERV-H Env and HERV-W Env, accompanied by increased seroreactivity
} Tomasz Brudek*1, Tove Christensen ${ }^{1}$, Lars Aagaard ${ }^{2}$, Thor Petersen ${ }^{3}$, Hans J Hansen ${ }^{3}$ and Anné Møller-Larsen ${ }^{1}$

\author{
Address: ${ }^{1}$ Department of Medical Microbiology and Immunology, University of Aarhus, DK-8000 Aarhus C, Denmark, ${ }^{2}$ Department of Molecular \\ Biology, University of Aarhus, DK-8000 Aarhus C, Denmark and ${ }^{3}$ Department of Neurology, University of Aarhus, DK-8000 Aarhus C, Denmark \\ Email: Tomasz Brudek* - tb@microbiology.au.dk; Tove Christensen - tc@microbiology.au.dk; Lars Aagaard - laa@daimi.au.dk; \\ Thor Petersen - thorpete@rm.dk; Hans J Hansen - hanshans@rm.dk; Anné Møller-Larsen - aml@microbiology.au.dk \\ * Corresponding author
}

Published: 16 November 2009

Retrovirology 2009, 6:104 doi:10.1186/1742-4690-6-104

This article is available from: http://www.retrovirology.com/content/6/1//04

(C) 2009 Brudek et al; licensee BioMed Central Ltd.

This is an Open Access article distributed under the terms of the Creative Commons Attribution License (http://creativecommons.org/licenses/by/2.0), which permits unrestricted use, distribution, and reproduction in any medium, provided the original work is properly cited.

\begin{abstract}
Background: The etiology of the neurogenerative disease multiple sclerosis (MS) is unknown. The leading hypotheses suggest that MS is the result of exposure of genetically susceptible individuals to certain environmental factor(s). Herpesviruses and human endogenous retroviruses (HERVs) represent potentially important factors in MS development. Herpesviruses can activate HERVs, and HERVs are activated in MS patients.
\end{abstract}

Results: Using flow cytometry, we have analyzed HERV-H Env and HERV-W Env epitope expression on the surface of PBMCs from MS patients with active and stable disease, and from control individuals. We have also analyzed serum antibody levels to the expressed HERV-H and HERV-W Env epitopes. We found a significantly higher expression of HERV-H and HERV-W Env epitopes on B cells and monocytes from patients with active MS compared with patients with stable MS or control individuals. Furthermore, patients with active disease had relatively higher numbers of $B$ cells in the PBMC population, and higher antibody reactivities towards HERV-H Env and HERV$W$ Env epitopes. The higher antibody reactivities in sera from patients with active MS correlate with the higher levels of HERV-H Env and HERV-W Env expression on B cells and monocytes. We did not find such correlations for stable MS patients or for controls.

Conclusion: These findings indicate that both HERV-H Env and HERV-W Env are expressed in higher quantities on the surface of B cells and monocytes in patients with active MS, and that the expression of these proteins may be associated with exacerbation of the disease.

\section{Background}

The cause of the inflammatory, neurodegenerative disease multiple sclerosis (MS) remains unknown. Etiological and epidemiological studies suggest that an infectious agent or agents operating on a background of genetic sus- ceptibility are probably involved in the pathogenesis [1]. Among the environmental factors human endogenous retroviruses (HERV) and the ubiquitously present herpesviruses are gaining growing attention, substantiated by an increasing number of reports suggesting their association 
with MS [2,3]. Recently, we have demonstrated increased cellular immune responses towards different herpesvirus and HERV antigens when they are concomitantly present in lymphocyte stimulation assays [4]. The cellular immune responses were synergistic in character and tended to be higher in MS patients in comparison with healthy controls.

This in vitro observation is pertinent only if herpesvirus and HERV antigens are concurrently present in vivo in MS patients. Herpesviruses are highly prevalent worldwide and they all cause latent infections that may subsequently become reactivated. HERVs are distributed in many copies throughout the human genome, and are inherited in a Mendelian fashion. Several herpesviruses are capable of HERV activation as previously demonstrated for HERV-K $[5,6]$ and HERV-W $[7,8]$. We have recently shown that the presence of inactivated herpesviruses can activate expression of HERVs in particle form in PBMCs from MS patients in vitro, most probably resulting in the concurrent presence of these two types of virus [9].

It has also been established that HERVs are present in activated form in vivo in MS patients. This is based on the demonstration of activated HERV-H $[10,11]$ and MSRV/ HERV-W [12,13] - virions - in blood from MS patients, and increased levels of HERV-H, HERV-K, and HERV-W RNA in MS brains [14]. HERV-W Env and Gag proteins have also been found in brain tissue from MS patients $[15,16]$. Our previous studies of humoral responses have demonstrated elevated levels of antibodies towards HERV-H Gag and Env epitopes in MS sera and cerebrospinal fluid (CSF) $[17,18]$, while others have reported antiMSRV/HERV-W antibodies in MS sera using a phage-display library of random pentadecapeptides as capture peptides [19]. These authors reported specific reactivity to four mimotopes in MS CSF. Two of these shared similarity with the HERV-W Env sequence [19]. However, we have subsequently found that all four mimotopes have higher similarities to HERV-H Env sequences [2]. Anti-HERV antibody reactivities will presumably be directed towards epitopes on virions as well as on lymphocyte surfaces.

In this manuscript, we present the first evidence that both HERV-H and HERV-W Envs are present at higher levels on the surface of PBMCs from patients with active or stable MS in comparison with PBMCs from healthy and neurological controls. Using flow cytometry, we have analyzed the levels of specific Env epitopes on the surface of different leukocyte populations. As a follow up to our previously published studies we have analyzed serum antibody reactivities towards these particular HERV-H and HERV-W Env epitopes, and correlated these reactivities with Env expression levels.

\section{Results \\ Western Blot and flow cytometric analyses of HERV-H Env and HERV-W Env expression on the surface of cells and particles obtained from MS cell cultures}

The polyclonal anti-HERV-H/-W Env TM (transmembrane region) and SU (surface unit region) rabbit antibodies were used in Western Blot analyses of purified retroviral particles from MS1946 cell culture, to detect whether these Env epitopes are present on virion surfaces.

The polyclonal anti-HERV-H/-W Env antibodies were raised towards equivalent but specific peptide epitopes: two peptides were localized in the TM regions of HERV-H and HERV-W Envs, respectively, and two peptides were localized in the SU regions. The results are presented in figure 1A. For HERV-H Env TM, a band of approximate molecular mass of $120 \mathrm{kDa}$ was present, whereas for HERV-H Env SU a band of approximate molecular mass of $60 \mathrm{kDa}$ was found. Bands of approximate molecular masses of $80 \mathrm{kDa}$, corresponding to both HERV-W Env TM and HERV-W Env SU, were present in virions produced by the MS1946 cell culture. The 60,80 and $120 \mathrm{kDa}$ bands were absent on blots incubated with the appropriate pre-immune sera. The bands at 60 and $80 \mathrm{kDa}$ are likely to correspond to monomeric glycosylated Envs, while the band at $120 \mathrm{kDa}$ may represent envelope protein aggregates or protein dimers as described for other retroviral Envs [20-23].

The differences in band sizes may be a result of HERV-H/ -W Env heterogenecity. At least three different ORFs for HERV-H Env, and one (Syncytin 1) for HERV-W Env, supplemented by a number of sequences with almost intact coding capacity, are dispersed in the human genome [2227].

To compare the presence of HERV Env epitopes on virions with expression of the same epitopes on cell surfaces of the virion producing cell culture, we performed flow cytometric analyses. The results presented in figure $1 \mathrm{~B}$ corroborate the Western Blot analyses as the cell culture expresses both HERV-H Env and HERV-W Env epitopes. However, whereas the equivalent HERV-W Env TM and SU epitopes are detectable on the surface of the cells, they are present at markedly lower levels than the HERV-H Env TM and SU epitopes.

\section{Flow cytometric analysis of HERV-H Env and HERV-W Env expression on PBMCs}

PBMCs isolated from patients with active MS, stable MS, from healthy controls and neurological non-inflammatory disease controls were incubated with the anti-HERVH/-W Env anti-sera (Additional File: Supplementary fig. 1 ), and also with anti-CD4, anti-CD8, anti-CD14, or antiCD19 antibodies, allowing a concurrent determination of 
A

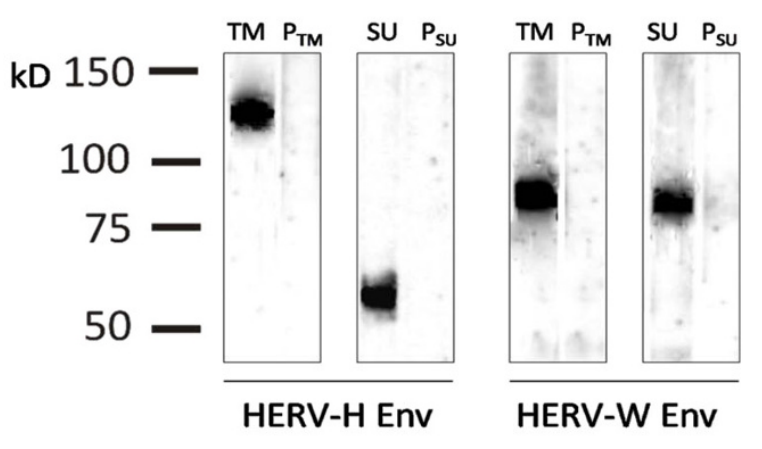

B

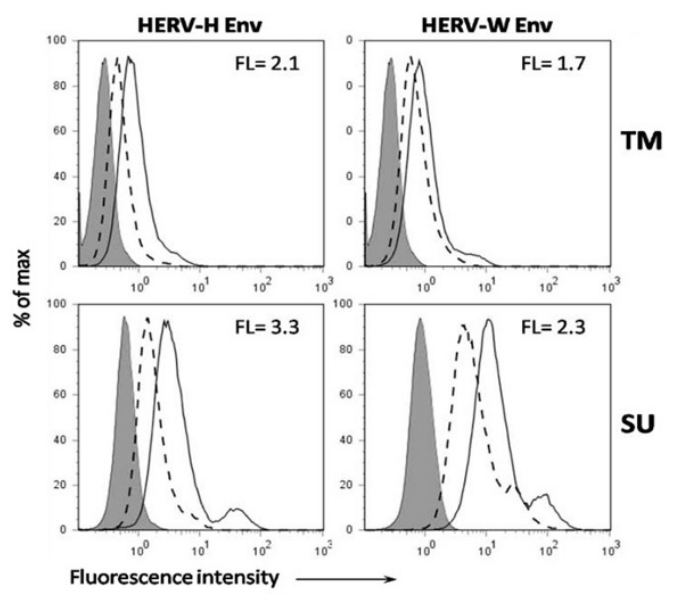

Figure I

Western blot analyses of OptiPrep purified HERV particles from MS 1946 long-term, lymphoblastoid cell cultures. Anti-HERV-H/-W Env TM and SU antibodies were raised in New Zealand white rabbits against 17-mer peptides localised at specific, but equivalent positions in the Env ORFs of HERV-H env62/HI 9 (Env HITM: aa489-505; Env H3SU: aa 370-386) and of syncytin I (Env WITM: aa4I543I, Env W3SU: aa301-317). Size markers are shown to the left. TM, SU -- anti-HERV Env TM/SU serum; $\mathrm{P}_{\mathrm{TM}}, \mathrm{P}_{\mathrm{SU}}$-appropriate pre-immune control sera. A. Flow cytometric analysis of surface expression of HERV-H Env and HERV-W Env TM and SU epitopes on cells from MS 1946 long-term lymphoblastoid cell cultures. The grey peaks represent the fluorescence of cells incubated with human lgG; the peaks with dashed line represent fluorescence of the cells incubated with pre-immune serum and FITC goat anti-rabbit antibodies; and the peaks with solid line represent fluorescence of the cells incubated with anti-HERV-H/-W Env TM/SU antisera and FITC goat anti-rabbit antibodies. Fluorescence indices are calculated as the ratio of the mean fluorescence of the cells incubated with anti-Env Abs to the mean fluorescence of the cells incubated with the appropriate control (pre-immune serum).
HERV Env expression, and determination of different leukocyte phenotypes.

The HERV-H Env and HERV-W Env TM and SU epitopes were also found to be present on CD19+ (B cells) as on CD14+ cells (monocytes), whereas we did not detect either HERV-H Env or HERV-W Env epitopes on CD4+ T cells or on CD8+ T cells (data not shown).

The surface expression of HERV-H Env TM epitopes on CD19+ cells was significantly higher in patients with MS, regardless of the disease activity, than in both groups of control individuals ( $p \leq 0.001)$ (fig. $2 \mathrm{~A}$ and fig. $2 \mathrm{C}$ ). The CD19+ cell expression of HERV-W Env TM epitopes was also significantly higher in both group of MS patients but only when compared with healthy controls $(p=0.02)$. CD14+ cell expression of HERV-H Env TM was significantly higher in both MS patient groups compared with neurological non-inflammatory disease controls $(p \leq$ 0.05).

Results obtained using anti-Env SU antibodies are presented in figure $2 \mathrm{~B}$ and 2D. HERV-H Env SU epitope expression was significantly higher on B cells and monocytes in all groups of individuals compared with HERV-W Env SU epitope expression $(p<0.01)$. The surface expression of the HERV-H Env SU epitope on CD19+ cells was significantly higher in patients with active MS than in patients with stable MS $(p=0.0001)$, healthy controls ( $p$ $=0.04)$, and neurological controls $(p=0.009)$. The CD19+ cell expression of the HERV-W Env SU epitope was significantly higher in the group of patients with active MS compared with stable MS $(p=0.0014)$, healthy controls $(p=0.0008)$, and neurological controls $(p=$ 0.0009). Similarly, on CD14+ cells HERV-H Env SU and HERV-W Env SU epitope expression levels were higher in patients with active MS compared with stable MS patients (for both SU epitopes, respectively $p=0.05, p=0.05$ ), healthy controls (for both SU epitopes, respectively $p=$ $0.03, p=0.0001$ ), and neurological controls (for both SU epitopes, respectively $p=0.05, p=0.0002$ ).

\section{Characterisation of leukocyte phenotypes}

We characterised the basic leukocyte populations in the PBMC samples from MS patients and healthy controls in parallel with the quantification of HERV-H/-W Env epitope expression on cell surfaces. The results are presented in figure 3.

Patients with active MS had a significantly higher number of B cells compared with patients with stable MS, healthy controls, or neurological non-inflammatory disease controls. We did not find significant differences in the numbers of CD4+ T cells, CD8+ T cells, or monocytes. 
A

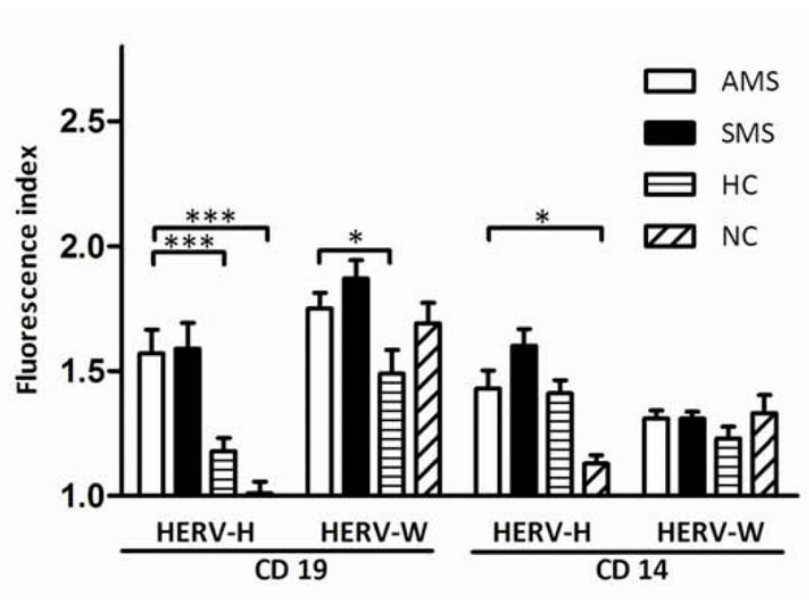

C
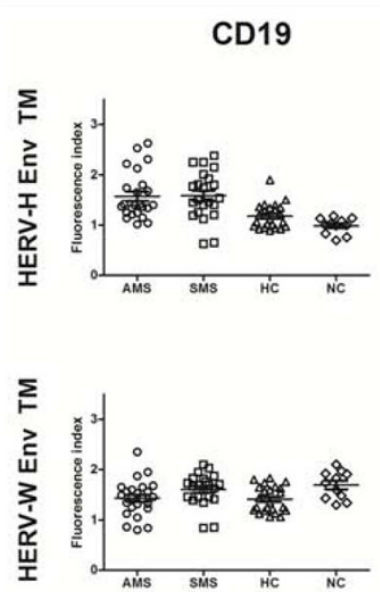

B

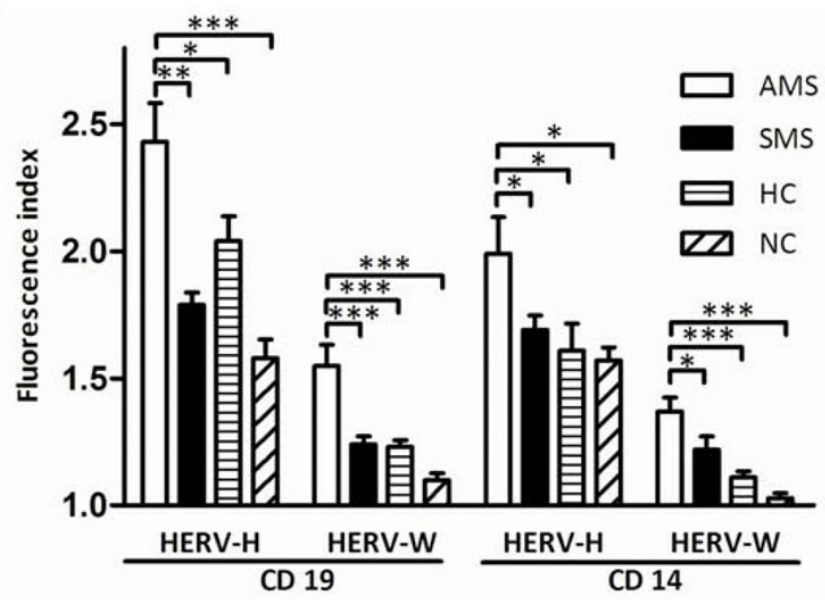

D

\section{CD19}

CD14
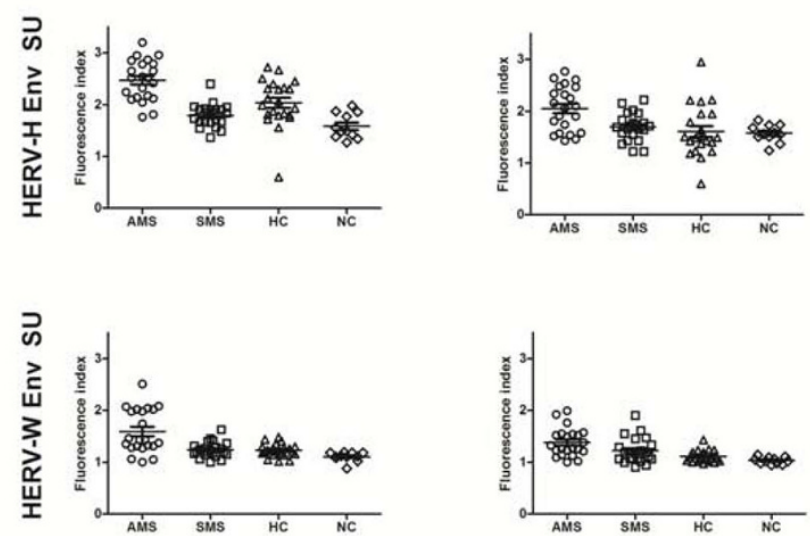

Figure 2

Flow cytometric analysis of surface expression of HERV-H Env and HERV-W Env on B-cells and monocytes from patients with active MS (AMS), stable MS patients (SMS), healthy individuals (HC), and neurological noninflammatory controls (NC). The results are presented as fluorescence indices calculated as the ratio of the mean fluorescence of the cells incubated with anti-Env Abs to the mean fluorescence of the cells incubated with the appropriate control (pre-immune serum). Mean values (2A) and scatter plots (2C) for cells incubated with anti-Env TM Abs. Mean values (2B) and scatter plots (2D) for cells incubated with anti-Env SU Abs. The standard errors for each group are presented. The significant differences $(P \leq 0.05)$ between the groups are shown. * $-P \leq 0.05$; ** $-P \leq 0.01$; ***.* $P \leq 0.00$ I on column bar graphs $2 A$ and $2 B$.

\section{Levels of anti-HERV-H Env and anti-HERV-W Env antibodies}

Having demonstrated that the surface expression of HERV-H/-W Env epitopes is higher on B cells and monocytes from patients with active MS, we analyzed the serological reactivity towards these epitopes. Accordingly, the peptides used for capture in this serological screening were identical to the peptides used for rabbit immunisation.

Figure 4 presents the analysis of the serological reactivity towards the HERV-H/-W Env peptide epitopes in sera 


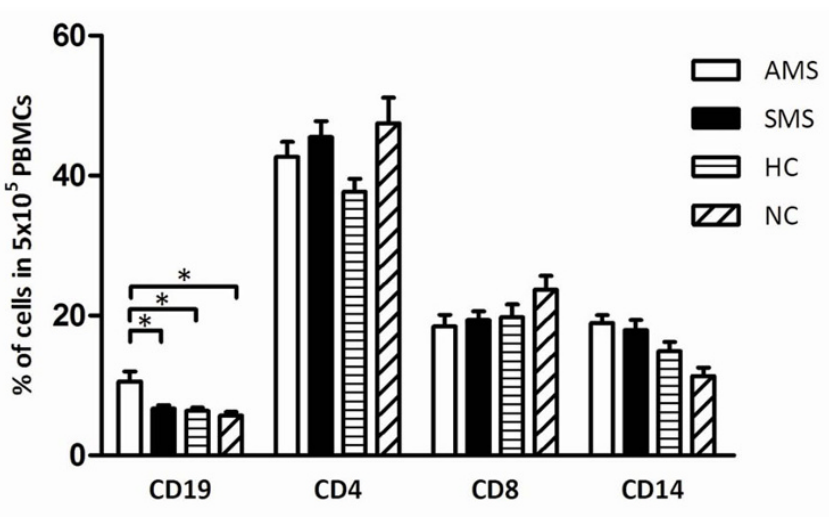

Figure 3

Flow cytometric analysis of different leukocyte populations in PBMCs isolated from patients with active MS (AMS), stable MS patients (SMS), healthy individuals (HC), and neurological non-inflammatory controls (NC). The bars represent the percentage of CDI9, CD4, CD8, and CDI4 cells in $5 \times 10^{6}$ PBMCs. The mean values and the standard error for each group are presented, and the significant differences $(P \leq 0.05)$ between the groups are shown. ${ }^{*}-P \leq 0.05$; ** $-P \leq 0.01$; ***. $P \leq 0.00$ I

from MS patients with active and stable MS as well as from healthy and disease controls. Sera from MS patients with active disease exhibited significantly higher levels of reactivity to all four peptides compared with patients with stable MS and with control individuals. Moreover, the levels of antibodies correlated with the levels of HERV-H/-W Env epitopes expressed on B cells and monocytes from patients with active MS, whereas such a correlation could not be found for stable MS patients, or for either group of control individuals (figure 5).

\section{Discussion}

An increasing number of reports indicate a role for HERVs in MS pathogenesis. The two Gammaretroviruses, HERV$\mathrm{H}$ and HERV-W are activated in MS patients during periods with disease activity $[10,13,17,18]$. Moreover, previous demonstrations of retroviral RNA in plasma/serum samples from MS patients indicate the presence of HERV virions and/or HERV proteins [10,13].

A previous study has analyzed HERV mRNA levels in brain and lymphocyte populations from $20 \mathrm{MS}$ patients unstratified for disease activity [28]. The present investigation is the first report of concomitant surface expression of both HERV-H Env and HERV-W Env epitopes on PBMCs from MS patients with active or stable MS, and controls. In addition, we have extended the findings by analyses of antibody reactivity towards these epitopes.
Initial investigations using flow cytometry demonstrated the presence of HERV-H Env and HERV-W Env on the surface of cells isolated from long-term MS cell cultures. Virions from the same cultures were analyzed by Western Blotting demonstrating the concomitant presence of both HERV-H and HERV-W Env epitopes in OptiPrep purified virions. This illustrates the known complexity of the HERV particles produced in MS [2]. Moreover, the different sizes of bands in Western Blots suggest individual Env expression patterns for HERV-H and HERV-W. In an earlier study we have demonstrated that Wistar rats, immunised with OptiPrep purified virions produced in longterm MS cell cultures, develop a serological response towards HERV-H Gag and Env derived synthetic peptides [17]. The present results are more direct indications of the presence of HERV Env epitopes on the virions produced in the MS cell cultures.

We also present analyses of the expression of HERV-H Env and HERV-W Env epitopes on the surface of PBMCs from patients with active MS, stable MS, and from control individuals. Both envelope proteins were detected on B cells and monocytes only, with the expression of HERV-H Env epitopes generally higher than the expression of HERV-W Env epitopes. Moreover, there were significantly higher quantities of both Envs on individual cells from patients with active MS compared with patients with stable MS and the control groups.

The apparent differences in the expression of HERV-H/-W Env TM and SU epitopes could be due to distinct features in the molecular Env-membrane interactions [29], or may be inherent in the assay as it could be due to differences in the glycosylation of the envelope proteins. Several of the HERV-H Env precursors are longer than the HERV-W Env (Syncytin 1) precursor and contain more putative $\mathrm{N}$ linked glycosylation sites $[20,22,23]$ which could affect the epitope exposure and thus its interaction with antibodies. An example is a putative glycosylation site at position aa370 in the HERV-H Env SU epitope, as it has previously been shown that glycosylation of HIV Env epitopes can affect their conformation as well as their interaction with antibodies [30].

Antibodies against TM region of HERV Envs have been successfully used in immune assays including flow cytometry and immunofluorescence staining by others, as they are able to detect the TM region of a given Env protein [3134]. Furthermore, it was shown for anti-HIV Env TM antibodies that they may utilize the cellular membrane to access and bind to gp41 [35], and that the membraneembedded HIV Envs elicit broader immune responses than soluble forms of Envs [36]. 

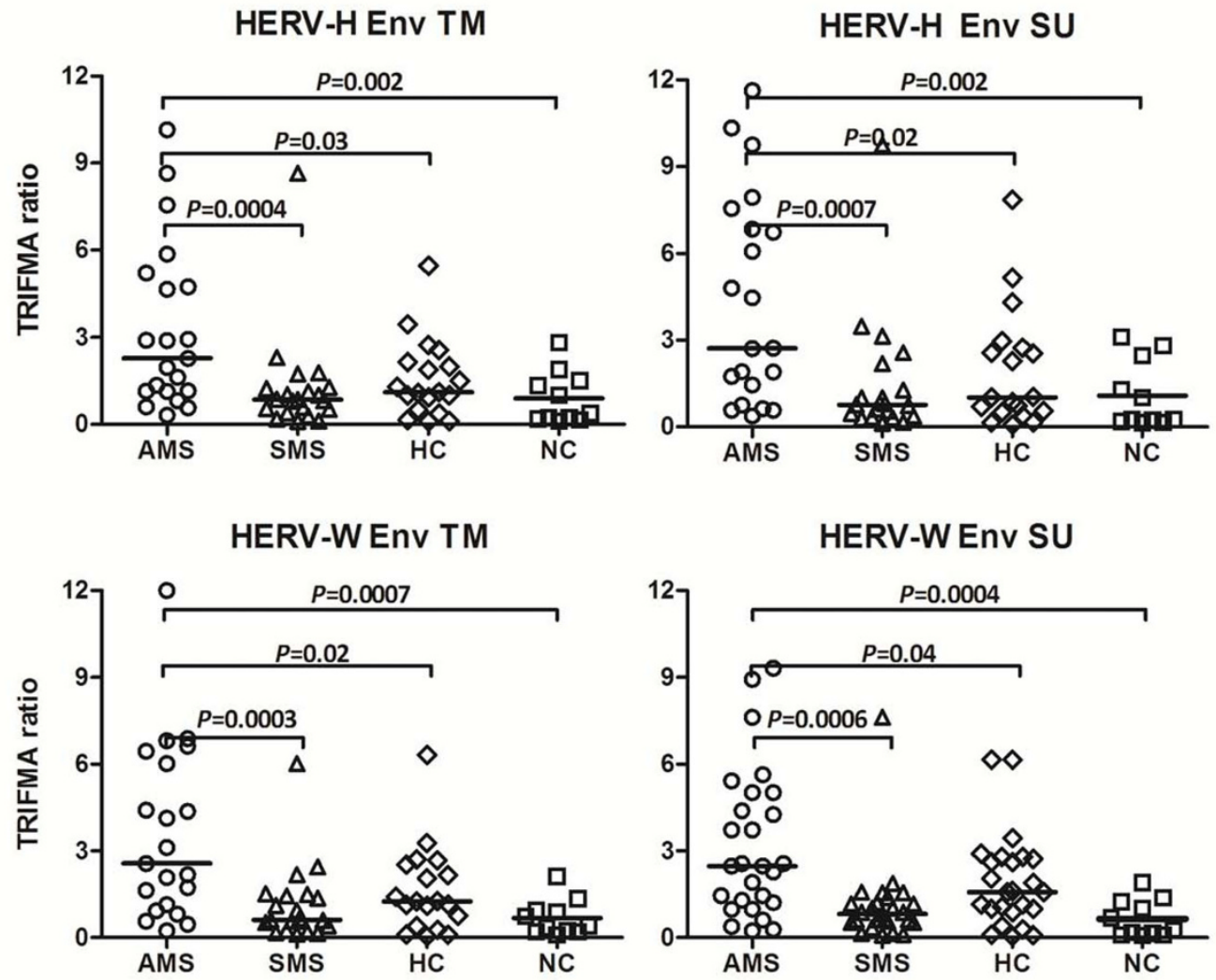

\section{Figure 4}

Seroreactivity to HERV-H Env and HERV-W Env derived peptides in patients with active MS (AMS), stable MS patients (SMS), and healthy individuals (HC) and neurological non-inflammatory controls (NC). The horizontal lines indicate median TRIFMA ratios for each group. Significant differences $(P \leq 0.05)$ between the groups are shown.

The expression of the Env epitopes exclusively on the surface of $\mathrm{B}$ cells and monocytes may be a consequence of the special relationship between HERVs and herpesviruses. It is already established that herpesviruses can activate HERVs [7-9,37-40] and it is likely that reactivated herpesviruses may transactivate HERVs at the transcriptional level. Many indications also exist that herpesviruses may be involved in MS pathogenesis. EBV, HHV-6, and VZV are the strongest candidates for this involvement [41]. It is well established that EBV persists within memory B cells [42], whereas monocytes are a site of latency for HHV-6 [43]. VZV DNA has been demonstrated in MS patient mononuclear cells in connection with disease exacerbation [44]. Noteworthy in this context is that we have previously shown that the concomitant presence of HERV and herpesvirus antigens induces synergistic cell-mediated immune responses $[4,45]$. It has also been demonstrated by others that patients with active MS have higher specific cellular immunity towards synthetic HERV Env TM peptides than patients with stable MS [46,47].
Concomitantly with the analyses of HERV Env expression, we analyzed the actual composition of the PBMC populations. Significantly higher numbers of B cells were present in the PBMCs from patients with active MS compared with healthy controls. Thus, the higher number of B cells together with the higher expression of HERV-H Env and HERV-W Env epitopes will augment the total amount of HERV Envs in active MS, while HERV Env expression is lower and hence may be down-regulated in patients with stable MS.

It is widely thought that T cells play a central role in MS pathogenesis and they have previously been the main focus of attention, since CD4+ T cells, as well as CD8+ T cells, reactive to myelin antigens, are present in MS patients and seem to be crucial in the development of some types of demyelinating lesions [48-53]. The autoreactive CD4+ T cells in MS may be activated in the periphery and once activated, they can cross the blood-brain barrier (BBB) [54,55]. Furthermore, several features of MS 
HERV-H Env SU
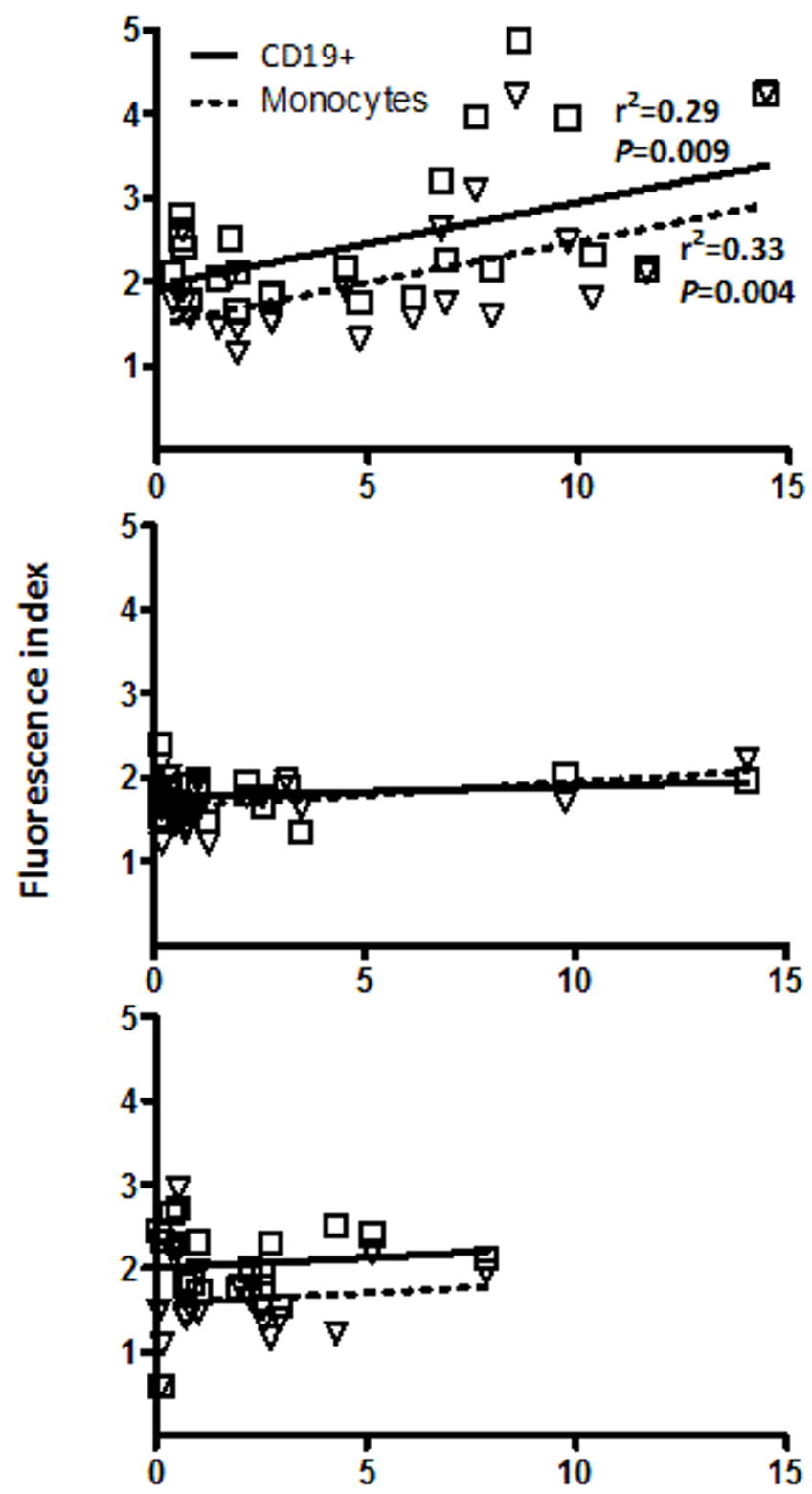

\section{HERV-W Env SU}
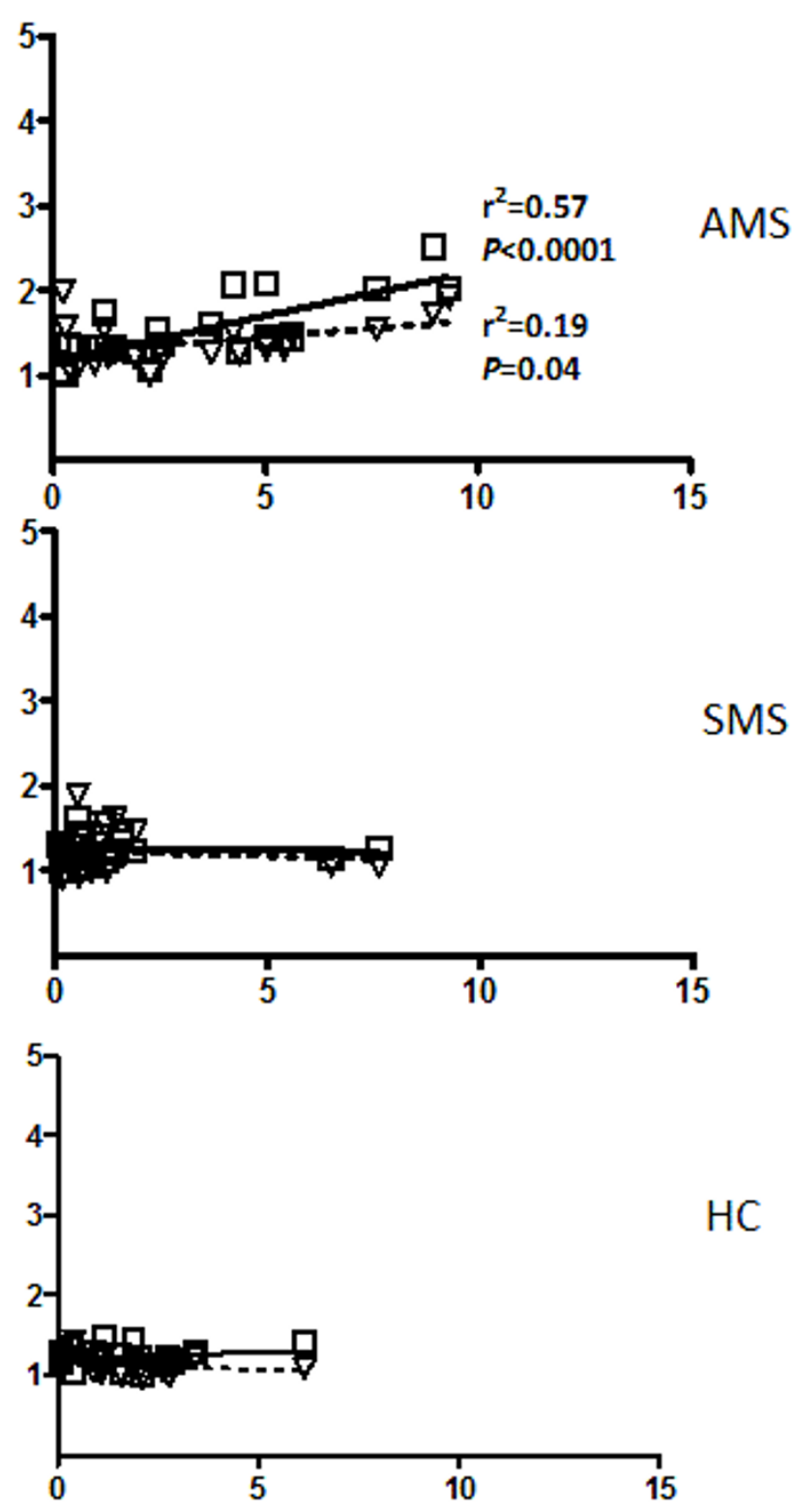

Figure 5

Multiparameter regression between the levels of anti-HERV-H/-W Env blood serum antibodies measured by TRIFMA vs. the levels of surface expression of HERV-H Env and HERV-W Env epitopes on B-cells and monocytes from patients with active MS (AMS), stable MS patients (SMS), and healthy individuals (HC) measured by flow cytometry (expressed as fluorescence indices calculated as the ratio of the mean fluorescence of the cells incubated with anti-Env Abs to the mean fluorescence of the cells incubated with the appropriate control (pre-immune serum)). The solid line for CDI9+ cells and dashed line for monocytes indicate the regression line. Correlation coefficient $\left(r^{2}\right)$ and statistical $(P \leq 0.05)$ significance are shown. 
lesions suggest that Th1 mediated immune responses play an important role in the inflammatory process [56]. A prominent expansion of CD8+ memory T cells have also been found in MS CSF and in MS brain tissue $[57,58]$. However, it is now emerging that other cell types, i.e. B cells, and also other factors are important [59-61]. Unlike activated T cells, $\mathrm{B}$ cells do not appear to cross the intact $\mathrm{BBB}$, whereas the occurrence of BBB damage in MS does permit the entry of $\mathrm{B}$ cells and antibodies into the CNS [62] presumably augmenting the characteristic intrathecal antibody synthesis found in a majority of MS patients. Furthermore, B-cell activation is associated with a more serious clinical outcome in MS [63], and B cells, plasma cells, and myelin-specific antibodies are present in some MS plaques [64-66]. One of the roles of the T-cells could be regulation of HERV expressing B cells. It is most likely that HERV expression stimulates antibody production, and in conjunction cytotoxic $\mathrm{T}$ cells and antibodies may act synergistically in reducing the increased HERV expression, thereby probably diminishing the immune reactivity and thereby also influence the pathogenesis of the actual MS attack.

The role of HERV-expressing monocytes is more uncertain and not extensively investigated, but besides being regulated by CD4 $+\mathrm{T}$ cells, these cells are antigen-presenting cells as are the $\mathrm{B}$ cells, which may contribute to T-cell reactivity towards the expressed HERV epitopes.

The apparent link between B-cell expansion in PBMCs and increased relapse-activity in MS is particularly interesting in view of the increasing awareness of the importance of $\mathrm{B}$ cells in MS pathogenesis (and the evident therapeutic potential in B-cell depletion [67]) although the main focus so far has been the B cells involved in intrathecal IgG synthesis in the CNS [68]. Our current findings are confirmed by reports of an increased number of B cells during MS relapses [61], and significantly increased levels of the B-cell survival promoter APRIL in MS patients [69]. Recently, short-lived plasma blasts were identified as the main effector B cell population involved in active inflammation in MS patients [70].

The elevated levels of HERV-H and HERV-W Env expression on B-cell and monocytes surfaces in samples from patients with active MS found in the present study is also closely reflected in the antibody response to HERV-H and HERV-W Env peptide epitopes. We have demonstrated significantly elevated levels of serum antibodies towards four representative Env peptide epitopes (HERV-H SU and TM, and HERV-W SU and TM) in samples from patients with active MS. Both the TM and SU regions of retroviral Envs are known to elicit serological responses [71], and whereas the amino acid sequences of these peptide epitopes clearly distinguish HERV-H Env from HERV-W Env, they are localised at equivalent positions in HERV-H and HERV-W Envs. This is completely consistent with our previous findings of increased antibody reactivities towards HERV-H/RGH-2 Env and Gag peptides associated with high MS disease activity compared with control groups such as patients with autoimmune diseases, patients with other neurological diseases, or healthy relatives of MS patients $[17,18]$. In the current study, serological activities actually correlate with the levels of HERV-H/ -W Env surface expression on B cells and monocytes. These consistent findings of higher anti-HERV antibody reactivities in the active phases of the disease substantiate a specific immune reactivity to HERVs in MS. Our findings may be paralleled in the chronic progressive, neurological disease HAM/TSP (HTLV-I (Human T-cell Leukaemia Virus) Associated Myelopathy/Tropical Spastic Paraparesis) which is caused by the human exogenous retrovirus HTLV-I. HAM/TSP is characterized by high levels of virusspecific cytotoxic $\mathrm{T}$ cells concomitantly with high levels of anti-HTLV-I Env antibodies in patient sera [72].

Apart from MS, HERVs have been implied in a number of other autoimmune disorders. Examples of suggested Gammaretroviral involvement in autoimmunity include HERV-E and HERV-W in psoriasis [31,73], HERV-E in systemic lupus erythematosus (SLE) [74,75], and HRES-1 in SLE [76,77]. The direct and/or indirect roles of HERV-H and HERV-W, the possible interactions between these two HERVs, and between HERVs and herpesviruses in MS, invites further investigations and there are several possible mechanisms by which HERVs could cause MS [2].

Our present results advocate the hypothesis that expressed ORFs from Gammaretroviruses such as HERV-H and HERV-W, as well as the complex interactions of HERVexpressing cells may play a role in MS development.

\section{Methods \\ Blood samples}

23 patients with active MS ( 18 females and 5 males, age $47 \pm 11$ years), 23 patients with stable MS (14 females and 9 males, age $49 \pm 15$ years), 22 healthy controls (11 females and 11 males, age $41 \pm 13$ years), and 11 patients with epilepsy ( 6 females and 5 males, age $45 \pm 13$ years) used as controls with an unrelated, neurological disease, were enrolled in the study. Patients with epilepsy comprise a heterogeneous group, often with focal inflammatory reactions as the cause of the seizures, which makes these patients a relevant control group for MS. Moreover, it has become increasingly evident that these inflammatory reactions mediate some of the changes seen in connection with seizures [78-81].

Stringent selection criteria were applied to all MS patients to ensure that only individuals with "typical" and clinically well-characterized relapsing-remitting MS were included in the study. The MS patients were selected at the 
Neurology Department, Aarhus University Hospital, and gave written informed consent to participate in the study. The Central Denmark Region Committee on Biomedical Research Ethics gave ethical approval for the sampling of blood, culturing of cells from patients, and use in the study. Information about the MS patients is provided in table 1. All MS patients fulfilled the diagnostic criteria of Poser et al., 1983 [82]. Relapsing-remitting (RR) MS is defined in accordance with the Poser criteria as at least two previous relapses in different CNS regions confirmed by neurological examination. Stratification of MS disease activity is also performed according to standard criteria: Active MS is defined as at least one relapse within one year prior to the examination (i.e. high annual relapse rate), while stable MS is defined as the absence of disease activity for at least a year as determined by standard clinical criteria. None of the MS patients or the control individuals had any evidence of an infectious disease within the last 3 months prior to the study.

Venous blood was drawn at the respective clinics and processed on the same day in our laboratory. PBMCs were prepared by standard Isopaque-Ficoll centrifugation. The separated cells were cryopreserved in RPMI with addition of $20 \%$ human serum (HS) and $10 \% \mathrm{DMSO}$, at $-135^{\circ} \mathrm{C}$ until use.

\section{Cell cultures}

The long-term, lymphoblastoid cell culture MS1946, originating from PBMCs from a patient with active MS, was grown as described previously $[83,84]$. In brief, the cells were grown at $0.5 \times 10^{6}$ cells $/ \mathrm{ml}$ of RPMI-1640, supplemented with $10 \%$ inactivated human serum. Cells were split three times a week and supplemented with fresh medium. Twenty four hours before harvest of supernatants, the suspensions were supplemented with additional fresh medium (approx. 30\% of the total volume) to obtain optimal growth conditions and thereby optimal virus production. Only batches with sufficiently high retrovirus production as confirmed by PERT (PCR-enhanced reverse transcriptase assay) for reverse transcriptase activity $[9,85]$ were used for virus purification.

\section{Anti-HERV Env Antibodies}

Polyclonal Anti-HERV-H Env and anti-HERV-W Env antibodies were raised in New Zealand White rabbits against 16-mer peptides localised at equivalent positions in the Env ORFs of HERV-H env62/H19 (Env H1TM: aa489505; Env H3SU: aa 370-386) [22,23] and syncytin 1 (Env W1TM: aa415-431, Env W3SU: aa301-317) [25], respectively. These peptide sequences fulfil the criteria of immunogenicity, and they are localised at equivalent positions in the HERV-H and HERV-W Envs, while having highly dissimilar amino acid sequences. Both the peptides and the anti-sera were prepared by Sigma-Genosys, UK. Preimmune sera were collected before immunisation. Two rabbits were immunised with each peptide, boosted 3 times, and after the final boost, peripheral blood was collected from each rabbit for subsequent measuring of anti-peptide antibodies.

Table I: Clinical data for MS patients: a -- active MS; RR -- relapsing-remitting; F -- female; M -- male.

\begin{tabular}{|c|c|c|c|c|c|c|c|}
\hline MS patient & MS type & Age (years) & Gender & MS patient & MS type & Age (years) & Gender \\
\hline I & $\mathrm{RR}$ a & 28 & $M$ & 24 & RR & 56 & $\mathrm{~F}$ \\
\hline 2 & $\mathrm{RR}$ a & 70 & $\mathrm{~F}$ & 25 & RR & 59 & $\mathrm{~F}$ \\
\hline 3 & $\mathrm{RR}$ a & 34 & $\mathrm{~F}$ & 26 & RR & 63 & $\mathrm{~F}$ \\
\hline 4 & RR a & 40 & $\mathrm{~F}$ & 27 & RR & 50 & $\mathrm{~F}$ \\
\hline 5 & $\mathrm{RR}$ a & 53 & $\mathrm{~F}$ & 28 & RR & 65 & $M$ \\
\hline 6 & $\mathrm{RR}$ a & 62 & $M$ & 29 & RR & 55 & $M$ \\
\hline 7 & $\mathrm{RR}$ a & 35 & $M$ & 30 & RR & 29 & $\mathrm{~F}$ \\
\hline 8 & $\mathrm{RR}$ a & 56 & $\mathrm{~F}$ & 31 & $\mathrm{RR}$ & 63 & $\mathrm{~F}$ \\
\hline 9 & $\mathrm{RR}$ a & 37 & $\mathrm{~F}$ & 32 & RR & 31 & $M$ \\
\hline 10 & $\mathrm{RR}$ a & 53 & $M$ & 33 & RR & 58 & $M$ \\
\hline 11 & $\mathrm{RR}$ a & 30 & $M$ & 34 & $\mathrm{RR}$ & 23 & $M$ \\
\hline 12 & RR a & 38 & $\mathrm{~F}$ & 35 & RR & 43 & $M$ \\
\hline 13 & $\mathrm{RR}$ a & 60 & $\mathrm{~F}$ & 36 & $\mathrm{RR}$ & 58 & $\mathrm{~F}$ \\
\hline 14 & $\mathrm{RR}$ a & 54 & $\mathrm{~F}$ & 37 & RR & 66 & $M$ \\
\hline 15 & RR a & 30 & $\mathrm{~F}$ & 38 & RR & 57 & $\mathrm{~F}$ \\
\hline 16 & $\mathrm{RR}$ a & 51 & $\mathrm{~F}$ & 39 & $\mathrm{RR}$ & 35 & $M$ \\
\hline 17 & $\mathrm{RR}$ a & 53 & $\mathrm{~F}$ & 40 & RR & 39 & $\mathrm{~F}$ \\
\hline 18 & $\mathrm{RR}$ a & 53 & $F$ & 41 & $\mathrm{RR}$ & 83 & $\mathrm{~F}$ \\
\hline 19 & $\mathrm{RR}$ a & 58 & $\mathrm{~F}$ & 42 & $\mathrm{RR}$ & 56 & $\mathrm{~F}$ \\
\hline 20 & RR a & 51 & $\mathrm{~F}$ & 43 & RR & 40 & $\mathrm{~F}$ \\
\hline 21 & $\mathrm{RR}$ a & 49 & $\mathrm{~F}$ & 44 & $\mathrm{RR}$ & 26 & $\mathrm{~F}$ \\
\hline 22 & $\mathrm{RR}$ a & 44 & $\mathrm{~F}$ & 45 & RR & 28 & $\mathrm{~F}$ \\
\hline 23 & RR a & 60 & $\mathrm{~F}$ & 46 & RR & 43 & $M$ \\
\hline
\end{tabular}


The specificity and cross-reactivity of the anti-HERV-H/-W Env antisera against the peptides were analyzed using TRIFMA assays. The anti-HERV-H Env epitope antisera were at least a 1000 times more reactive towards the HERV-H Env peptides than towards HERV-W Env peptides, and vice versa (data not shown).

\section{Virion purification and Western Blotting}

The expression of HERV-H and HERV-W Env epitopes on the virions produced by the long-term MS1946 cell culture was analysed by Western Blotting of purified particles. These virions were purified by ultracentrifugation of $800 \mathrm{ml}$ samples of cell culture supernatants $\left(1.2 \times 10^{6}\right.$ cells $/ \mathrm{ml}$ ) in self-generating Iodixanol gradients (Nycomed, Norway) as described in detail elsewhere [86], and fractionated. Fractions with high reverse transcriptase activity as measured by PERT were pooled, suspended in TNE $(50 \mathrm{mM}$ Tris-HCl pH 7.5, $100 \mathrm{mM} \mathrm{NaCl}, 1 \mathrm{mM}$ EDTA) with $0.1 \%$ HSA (human serum albumin) and stored at $-70^{\circ} \mathrm{C}$ until use.

The pooled virion-containing gradient fractions were loaded onto $4-12 \%$ Bis-Tris precast gels, electrophoresed in MOPS buffer (Criterion TM XT system, Biorad, Richmond, CA, USA), and electrophoretically transferred to Hybond nitrocellulose membranes in transferbuffer (25 $\mathrm{mM}$ Tris-HCl, $192 \mathrm{mM}$ Glycine, 20\% EtOH, 0,1\% SDS, $\mathrm{pH}$ 8.5). Relative molecular sizes were interpolated from curves constructed on the basis of coloured marker proteins (Biorad Richmond, CA, USA, Precision standard). After incubation with the primary, polyclonal rabbit antibodies (diluted 1/2500) at room temperature overnight, the blots were treated with horseradish peroxidaselabeled secondary goat anti-rabbit antibodies (diluted 1/ 4000)(DAKO, Denmark) followed by enhanced chemiluminescence reagent (Super Signal West Pico, Pierce Biotechnologies, Rockville, USA). Blots were visualised using a Kodak ID Image Station.

\section{Flow Cytometric Analysis}

Multi-colour flow cytometry was performed to determine both the phenotypes of the cells, and HERV-H Env and/or HERV-W Env cell surface expression. The phenotypes were determined using monoclonal antibodies, anti-CD19-PE (cat.no. 12-0199), anti-CD4-PE-Cy5 (cat.no. 15-0049), anti-CD8-PE-Cy7 (cat.no. 25-0088), and anti-CD14-PECy7 (cat.no. 25-0149) purchased from eBioscience. The anti-HERV-H Env and anti-HERV-W Env antibodies described above were used for HERV-H and HERV-W Env epitope detection, visualised using goat anti-rabbit IgG, $\mathrm{F}\left(\mathrm{ab}^{\prime}\right)_{2}$ conjugated with FITC (PIERCE, cat. no. 31573). Isotype controls included mouse IgG1PE, PE-Cy5, and PECy7 (eBioscience cat. no. 12-4714, 15-4714, 25-4714). Pre-immune sera from the appropriate rabbits were used as controls for the anti-Env antibodies. The monoclonal antibodies were used in concentrations as suggested by the manufacturer. The polyclonal rabbit antibodies were diluted 1/1000 before use. Prior to staining with antibodies all PBMC samples were incubated with human IgG (Statens Serum Institute, Beriglobin, cat. no. 2948) in a concentration of $100 \mu \mathrm{g} / 10^{6} \mathrm{cells} / \mathrm{ml}$ to avoid non-specific antibody binding.

Flow cytometry analysis was performed on a Beckman Coulter Cytomics FC500 flow cytometer. The data were analyzed using Flow-Jo v.7 software (Treestar, San Carlos, CA, USA). A total of at least 50,000 events were analyzed for each sample.

The results from the relative quantification of HERV-H Env and HERV-W Env epitope expression are presented as fluorescence (FL) indices, which were obtained by dividing the mean fluorescence of the cells incubated with antiEnv antibodies by the mean fluorescence of cells incubated with the appropriate pre-immune control serum.

\section{Time-Resolved Immunofluorometric Assay (TRIFMA) for anti- Env peptide antibodies}

TRIFMA is a highly sensitive and reliable method for antibody detection, using europium-labelled secondary antibodies. The peptides used for capture were the same as the peptides used for raising the polyclonal anti-HERV-H Env and anti-HERV-W Env rabbit antibodies. The assay was performed essentially as described previously $[17,18]$. In brief, sera were diluted 1/500 in TBS/Tween and tested in duplicate. The fluorescence was measured using a timeresolved plate fluorometer (LKB, Wallac). Results are presented as TRIFMA ratios, defined as individual measurements in relation to the mean of TRIFMA controls. As TRIFMA inter-assay controls, 8 sera from healthy individuals representing both high and low responders were used [18]. The control sera were included in all TRIFMA assays.

\section{Statistical analysis}

For statistical calculations, Mann-Whitney testing and multiple regression testing were performed using GraphPad Instat ver.3. For TRIFMA, the highest and the lowest responder were excluded from each group before the analysis was performed.

\section{Competing interests}

The authors declare that they have no competing interests.

\section{Authors' contributions}

TB has made substantial contributions to conception, design, and acquisition of data, as well as statistical analysis and interpretation of data. TB carried out the molecular genetic studies, Western Blotting, flow cytometric analyses, and TRIFMA immunoassays. TB wrote a first draft of the paper, with contributions from other authors 
to the following drafts and the final version. TB has given final approval of the version to be published. TC has made substantial contributions to conception, design, analysis, and interpretation of data. TC has been involved in drafting the manuscript and revising it critically for important intellectual content. TC has given final approval of the version to be published.

LA has made substantial contributions to conception, design, analysis and interpretation of data from molecular genetic studies. LA has given final approval of the version to be published. TP has made substantial contributions to conception of the study. TP has been responsible for enrolment of MS patients and collection of blood samples. TP has given final approval of the version to be published.

HJH has made substantial contributions to conception of the study. HJH has been responsible for enrolment of MS patients and collection of blood samples. HJH has given final approval of the version to be published. AML has conceived and has been the main coordinator of the study. AML has made substantial contributions to conception, design, analysis, and interpretation of data. AML has been involved in drafting the manuscript and revising it critically for important intellectual content. AML has given final approval of the version to be published. All authors read and approved the final manuscript.

\section{Additional material}

\section{Additional file 1}

Supplementary figure 1. Flow cytometric analysis of surface expression of HERV-H and HERV-W Env epitopes on B-cells and monocytes from patients with active MS (AMS), stable MS patients (SMS), healthy individuals (HC), and neurological non-inflammatory controls (NC). The figure is a presentation of flow cytometric data analyses. From each group, two individuals representing high and low levels of HERV-H/-W Env epitope expression are shown. Blue peak - cells incubated with secondary goat anti-rabbit IgG, F(ab') ${ }_{2}$ FITC; green peak - cells incubated with appropriate pre-immune serum; red peak - cells incubated with antiHERV-H/W Env TM/SU serum. The numbers in square brackets represent the fluorescence index calculated as the ratio of the mean fluorescence of the cells incubated with anti-Env Abs to the mean fluorescence of the cells incubated with the appropriate control (pre-immune serum).

Click here for file

[http://www.biomedcentral.com/content/supplementary/17424690-6-104-S1.PPT]

\section{Acknowledgements}

We gratefully acknowledge the following sponsors for their financial support: The Carlsberg Foundation, The Augustinus Foundation; The Beckett Foundation; The Aarhus University Research Foundation; The Danish Multiple Sclerosis Society; Aase and Einar Danielsen's Foundation; Ingeborg og Leo Dannins Fond for Videnskabelig Forskning; Johnsen og Hustru's Mindelegat; The Danish Medical Research Council; Goof Fonden; Fonden til
Lægevidenskabens Fremme; Jascha Fonden; Direktør Jacob Madsens Fond; Torben og Alice Frimodts Fond; Vilhelm Bangs Fond; C.C. Klestrups Fond, Dagmar Marshalls Fond, Oticon Fonden and The Faculty of Health Science, Aarhus University. We express our sincere thanks to Vivi Brandt, Margit Aagaard, Lisbeth Jensen and Ruth Nielsen for their excellent technical assistance.

\section{References}

I. Noseworthy $\mathrm{JH}$ : Progress in determining the causes and treatment of multiple sclerosis. Nature 1999, 399:A40-A47.

2. Christensen T: Association of human endogenous retroviruses with multiple sclerosis and possible interactions with herpes viruses. Rev Med Virol 2005, 15: 179-2II.

3. Simmons A: Herpesvirus and multiple sclerosis. Herpes 200I, 8:60-63.

4. Brudek T, Christensen T, Hansen HJ, Bobecka J, Moller-Larsen A: Simultaneous presence of endogenous retrovirus and herpes virus antigens has profound effect on cell-mediated immune responses: implications for multiple sclerosis. AIDS Res Hum Retroviruses 2004, 20:415-423.

5. Sutkowski N, Conrad B, Thorley-Lawson DA, Huber BT: EpsteinBarr Virus Transactivates the Human Endogenous Retrovirus HERV-KI 8 that Encodes a Superantigen. Immunity 200I, I5:579-589.

6. Sutkowski N, Chen G, Calderon G, Huber BT: Epstein-Barr virus latent membrane protein LMP-2A is sufficient for transactivation of the human endogenous retrovirus HERV-KI8 superantigen. J Virol 2004, 78:7852-7860.

7. Ruprecht K, Obojes K, Wengel V, Gronen F, Kim KS, Perron H, Schneider-Schaulies J, Rieckmann P: Regulation of human endogenous retrovirus $W$ protein expression by herpes simplex virus type I: implications for multiple sclerosis. I Neurovirol 2006, | 2:65-7|.

8. Perron H, Suh M, Lalande B, Gratacap B, Laurent A, Stoebner P, Seigneurin JM: Herpes simplex virus ICPO and ICP4 immediate early proteins strongly enhance expression of a retrovirus harboured by a leptomeningeal cell line from a patient with multiple sclerosis. J Gen Virol I993, 74(Pt I):65-72.

9. Brudek T, Luhdorf P, Christensen T, Hansen HJ, Moller-Larsen A: Activation of endogenous retrovirus reverse transcriptase in multiple sclerosis patient lymphocytes by inactivated HSV-I, HHV-6 and VZV. J Neuroimmunol 2007, 187:147-155.

10. Christensen T, Dissing SP, Riemann H, Hansen HJ, Moller-Larsen A: Expression of sequence variants of endogenous retrovirus RGH in particle form in multiple sclerosis. Lancet 1998, 352: 1033 .

II. Christensen T, Dissing SP, Riemann H, Hansen HJ, Munch M, Haahr $S$, Moller-Larsen A: Molecular characterization of HERV-H variants associated with multiple sclerosis. Acta Neurologica Scandinavica 2000, I01:229-238.

12. Perron H, Garson JA, Bedin F, Beseme F, Paranhos-Baccala G, Komurian-Pradel F, Mallet F, Tuke PW, Voisset C, Blond JL, Lalande B, Seigneurin JM, Mandrand $B$ : Molecular identification of a novel retrovirus repeatedly isolated from patients with multipleásclerosis. PNAS 1997, 94:7583-7588.

13. Garson JA, Tuke PW, Giraud P, Paranhos-Baccala G, Perron H: Detection of virion-associated MSRV-RNA in serum of patients with multiple sclerosis. Lancet 1998, 35 I:33.

14. Johnston JB, Silva C, Holden J, Warren KG, Clark AW, Power C: Monocyte activation and differentiation augment human endogenous retrovirus expression: implications for inflammatory brain diseases. Ann Neurol 2001, 50:434-442.

15. Perron H, Lazarini F, Ruprecht K, Pechoux-Longin C, Seilhean D, Sazdovitch V, Creange A, Battail-Poirot N, Sibai G, Santoro L, Jolivet M, Darlix JL, Rieckmann P, Arzberger T, Hauw JJ, Lassmann H: Human endogenous retrovirus (HERV)-W ENV and GAG proteins: Physiological expression in human brain and pathophysiological modulation in multiple sclerosis lesions. Journal of NeuroVirology 2005, I I:23-33.

16. Mameli G, Astone V, Arru G, Marconi S, Lovato L, Serra C, Sotgiu S, Bonetti B, Dolei A: Brains and peripheral blood mononuclear cells of multiple sclerosis (MS) patients hyperexpress MSassociated retrovirus/HERV-W endogenous retrovirus, but not Human herpesvirus 6. J Gen Virol 2007, 88:264-274. 
17. Christensen T, Sorensen PD, Hansen HJ, Moller-Larsen A: Antibodies against a human endogenous retrovirus and the preponderance of env splice variants in multiple sclerosis patients. Mult Scler 2003, 9:6-I5.

18. Christensen T, Petersen T, Thiel S, Brudek T, Ellermann-Eriksen S, Moller-Larsen A: Gene-environment interactions in multiple sclerosis: Innate and adaptive immune responses to human endogenous retrovirus and herpesvirus antigens and the lectin complement activation pathway. Journal of Neuroimmunology 2007, I 83: I75-188.

19. Jolivet-Reynaud C, Perron H, Ferrante P, Becquart L, Dalbon P, Mandrand B: Specificities of Multiple Sclerosis Cerebrospinal Fluid and Serum Antibodies against Mimotopes. Clinical Immunology 1999, 93:283-293.

20. Blond JL, Beseme F, Duret L, Bouton O, Bedin F, Perron H, Mandrand B, Mallet F: Molecular Characterization and Placental Expression of HERV-W, a New Human Endogenous Retrovirus Family. J Virol 1999, 73: I I75-I I85.

21. Blond JL, Lavillette D, Cheynet V, Bouton O, Oriol G, Chapel-Fernandes S, Mandrand B, Mallet F, Cosset FL: An Envelope Glycoprotein of the Human Endogenous Retrovirus HERV-W Is Expressed in the Human Placenta and Fuses Cells Expressing the Type D Mammalian Retrovirus Receptor. J Virol 2000, 74:3321-3329.

22. Lindeskog M, Mager DL, Blomberg J: Isolation of a Human Endogenous Retroviral HERV-H Element with an Open env Reading Frame. Virology 1999, 258:44|-450.

23. de Parseval N, Casella JF, Gressin L, Heidmann T: Characterization of the Three HERV-H Proviruses with an Open Envelope Reading Frame Encompassing the Immunosuppressive Domain and Evolutionary History in Primates. Virology 200 I 279:558-569.

24. de Parseval N, Lazar V, Casella JF, Benit L, Heidmann T: Survey of Human Genes of Retroviral Origin: Identification and Transcriptome of the Genes with Coding Capacity for Complete Envelope Proteins. J Virol 2003, 77:104|4-10422.

25. Mallet F, Bouton O, Prudhomme S, Cheynet V, Oriol G, Bonnaud B Lucotte G, Duret L, Mandrand $B$ : The endogenous retroviral locus ERVWEI is a bona fide gene involved in hominoid placental physiology. PNAS 2004, 101:1731-1736.

26. Voisset C, Bouton O, Bedin F, Duret L, Mandrand B, Mallet F, Paranhos-Baccala G: Chromosomal distribution and coding capacity of the human endogenous retrovirus HERV-W family. AIDS Res Hum Retroviruses 2000, 16:731-740.

27. Villesen $P$, Aagaard L, Wiuf C, Pedersen F: Identification of endogenous retroviral reading frames in the human genome. Retrovirology 2004, I:32.

28. Antony JM, Izad M, Bar-Or A, Warren KG, Vodjgani M, Mallet F, Power C: Quantitative Analysis of Human Endogenous Retrovirus-W env in Neuroinflammatory Diseases. AIDS Res Hum Retroviruses 2006, 22: I253-1259.

29. Costin J: Cytopathic Mechanisms of HIV-I. Virology Journal 2007, 4:100.

30. Huang X, Barchi JJ, Lung FDT, Roller PP, Nara PL, Muschik J, Garrity RR: Glycosylation Affects both the Three-Dimensional Structure and Antibody Binding Properties of the HIV-IIIIB GP I 20 Peptide RP I35. Biochemistry | 997, 36: | 0846- I0856.

31. Bessis D, Moles JP, Basset-Seguin N, Tesniere A, Arpin C, Guilhou J]: Differential expression of a human endogenous retrovirus $E$ transmembrane envelope glycoprotein in normal, psoriatic and atopic dermatitis human skin. British Journal of Dermatology 2004, I 5 1:737-745.

32. Buscher K, Hahn S, Hofmann M, Trefzer U, Ozel M, Sterry W, Lower J, Lower R, Kurth R, Denner J: Expression of the human endogenous retrovirus-K transmembrane envelope, Rec and Np9 proteins in melanomas and melanoma cell lines. [Article]. Melanoma Research 2006, 16:223-234

33. Cheynet V, Ruggieri A, Oriol G, Blond JL, Boson B, Vachot L, Verrier $B$, Cosset FL, Mallet F: Synthesis, assembly, and processing of the Env ERVWEI/syncytin human endogenous retroviral envelope. J Virol 2005, 79:5585-5593.

34. Mangeney $M$, Heidmann $T$ : Tumor cells expressing a retroviral envelope escape immune rejection in vivo. Proc Natl Acad Sci USA 1998, 95:14920-14925.

35. Veiga AS, Pattenden LK, Fletcher JM, Castanho MA, Aguilar MI: Inter actions of HIV-I antibodies $2 F 5$ and 4 EIO with a gp4I epitope prebound to host and viral membrane model systems. Chembiochem 2009, I 0:1032-1044.

36. McBurney SP, Young KR, Ross TM: Membrane embedded HIV-I envelope on the surface of a virus-like particle elicits broader immune responses than soluble envelopes. Virology 2007, 358:334-346.

37. Kwun HJ, Han HJ, Lee WJ, Kim HS, Jang KL: Transactivation of the human endogenous retrovirus $K$ long terminal repeat by herpes simplex virus type I immediate early protein 0 . Virus Res 2002, 86:93-100.

38. Lee WJ, Kwun HJ, Kim HS, Jang KL: Activation of the human endogenous retrovirus $\mathrm{W}$ long terminal repeat by herpes simplex virus type I immediate early protein I. Mol Cells 2003, 1 5:75-80.

39. Perre $\mathrm{P}$ Van de, Segondy $M$, Foulongne $\mathrm{V}$, Ouedraogo A, Konate I, Huraux JM, Mayaud P, Nagot N: Herpes simplex virus and HIV. I: deciphering viral synergy. The Lancet Infectious Diseases 2008, 8:490-497.

40. Hsiao FC, Tai AK, Deglon A, Sutkowski N, Longnecker R, Huber BT: EBV LMP-2A employs a novel mechanism to transactivate the HERV-KI 8 superantigen through its ITAM. Virology 2009, 385:26I-266.

4I. Christensen T: Human herpesviruses in MS. Int MS / 2007 , | 4:4 |-47.

42. Young LS, Rickinson AB: Epstein-Barr virus: $\mathbf{4 0}$ years on. Nat Rev Cancer 2004, 4:757-768.

43. Kondo K, Kondo T, Okuno T, Takahashi M, Yamanishi K: Latent human herpesvirus 6 infection of human monocytes/macrophages. J Gen Virol 1991, 72:140|-|408.

44. Ordonez G, Pineda B, Garcia-Navarrete R, Sotelo J: Brief Presence of Varicella-zoster Viral DNA in Mononuclear Cells During Relapses of Multiple Sclerosis. Arch Neurol 2004, 6 I:529-532.

45. Brudek T, Christensen T, Hansen HJ, Petersen T, Moller-Larsen A: Synergistic immune responses induced by endogenous retrovirus and herpesvirus antigens result in increased production of inflammatory cytokines in multiple sclerosis patients. Scand I Immunol 2008, 67:295-303.

46. Trabattoni D, Ferrante P, Fusi ML, Saresella M, Caputo D, Urnovitz H, Cazzullo CL, Clerici M: Augmented type I cytokines and human endogenous retroviruses specific immune responses in patients with acute multiple sclerosis. I Neurovirol 2000 , 6(Suppl 2):S38-S4I.

47. Clerici M, Fusi ML, Caputo D, Guerini FR, Trabattoni D, Salvaggio A, Cazzullo CL, Arienti D, Villa ML, Urnovitz HB, Ferrante P: Immune responses to antigens of human endogenous retroviruses in patients with acute or stable multiple sclerosis. Journal of $\mathrm{Neu}$ roimmunology 1999, 99:173-182.

48. Bielekova B, Sung MH, Kadom N, Simon R, McFarland H, Martin R: Expansion and Functional Relevance of High-Avidity MyelinSpecific CD4+ T Cells in Multiple Sclerosis. J Immunol 2004, I 72:3893-3904.

49. Hong J, Zang YC, Li S, Rivera VM, Zhang JZ: Ex vivo detection of myelin basic protein-reactive $T$ cells in multiple sclerosis and controls using specific TCR oligonucleotide probes. Eur J Immunol 2004, 34:870-88I.

50. Olsson T, Sun J, Hillert J, Hojeberg B, Ekre HP, Andersson G, Olerup $\mathrm{O}$, Link $\mathrm{H}$ : Increased numbers of $T$ cells recognizing multiple myelin basic protein epitopes in multiple sclerosis. Eur J Immunol 1992, 22: 1083-1087.

5I. Olsson T, Zhi WW, Hojeberg B, Kostulas V, Jiang YP, Anderson G, Ekre HP, Link H: Autoreactive T lymphocytes in multiple sclerosis determined by antigen-induced secretion of interferongamma. J Clin Invest 1990, 86:981-985.

52. Lassmann H, Ransohoff RM: The CD4-Th I model for multiple sclerosis: a critical [correction of crucial] re-appraisal. Trends Immunol 2004, 25: I32-I37.

53. Zang YCQ, Li S, Rivera VM, Hong J, Robinson RR, Breitbach WT, Killian J, Zhang JZ: Increased CD8+ Cytotoxic T Cell Responses to Myelin Basic Protein in Multiple Sclerosis. J Immunol 2004, I 72:5|20-5 I 27

54. Sheremata WA, Minagar A, Alexander JS, Vollmer T: The role of alpha-4 integrin in the aetiology of multiple sclerosis: current knowledge and therapeutic implications. CNS Drugs 2005, 19:909-922.

55. Archelos J], Previtali SC, Hartung HP: The role of integrins in immune-mediated diseases of the nervous system. Trends Neurosci 1999, 22:30-38. 
56. Merrill JE: Proinflammatory and antiinflammatory cytokines in multiple sclerosis and central nervous system acquired immunodeficiency syndrome. J Immunother 1992, I 2:167-I 170.

57. Jacobsen M, Cepok S, Quak E, Happel M, Gaber R, Ziegler A, Schock $\mathrm{S}$, Oertel WH, Sommer N, Hemmer B: Oligoclonal expansion of memory CD8+ $T$ cells in cerebrospinal fluid from multiple sclerosis patients. Brain 2002, I 25:538-550.

58. Skulina C, Schmidt S, Dornmair K, Babbe H, Roers A, Rajewsky K, Wekerle H, Hohlfeld R, Goebels N: Multiple sclerosis: Brain-infiltrating CD8+ T cells persist as clonal expansions in the cerebrospinal fluid and blood. PNAS 2004, 101 :2428-2433.

59. Duddy M, Bar-Or A: B-cells in multiple sclerosis. Int MS J 2006 13:84-90.

60. Antel J, Bar-Or A: Roles of immunoglobulins and B cells in multiple sclerosis: From pathogenesis to treatment. Journal of Neuroimmunology 2006, 180:3-8.

61. Paz A, Fiszer U, Zaborski J, Korlak J, Czlonkowski A, Czlonkowska A: Phenotyping analysis of peripheral blood leukocytes in patients with multiple sclerosis. European Journal of Neurology 1999, 6:347-352.

62. Zhang Y, Da RR, Guo W, Ren HM, Hilgenberg LG, Sobel RA, Tourtellotte WW, Smith MA, Olek M, Gupta S, Robertson RT, Nagra R, Noort S Van Den, Qin Y: Axon Reactive B Cells Clonally Expanded in the Cerebrospinal Fluid of Patients with Multiple Sclerosis. Journal of Clinical Immunology 2005, 25:254-264.

63. Petereit HF, Reske D: Expansion of antibody reactivity in the cerebrospinal fluid of multiple sclerosis patients - follow-up and clinical implications. Cerebrospinal Fluid Research 2005, 2:3

64. Esiri MM: Immunoglobulin-containing cells in multiple-sclerosis plaques. Lancet 1977, 2:478.

65. Genain CP, Cannella B, Hauser SL, Raine CS: Identification of autoantibodies associated with myelin damage in multiple sclerosis. Nat Med 1999, 5: I70-175

66. Mattson DH, Roos RP, Arnason BGW: Isoelectric focusing of IgG eluted from multiple sclerosis and subacute sclerosing panencephalitis brains. Nature 1980, 287:335-337.

67. Bennett JL, Stuve O: Update on inflammation, neurodegeneration, and immunoregulation in multiple sclerosis: therapeutic implications. Clin Neuropharmacol 2009, 32: I2I-I 32.

68. Fraussen J, Vrolix K, Martinez-Martinez P, Losen M, De Baets MH, Stinissen $\mathrm{P}$, Somers V: B cell characterization and reactivity analysis in multiple sclerosis. Autoimmunity Reviews 2009, 8:654-658.

69. Thangarajh M, Masterman T, Hillert J, Moerk S, Jonsson R: A Proliferation-inducing Ligand (APRIL) is Expressed by Astrocytes and is Increased in Multiple Sclerosis. Scandinavian Journal of Immunology 2007, 65:92-98.

70. Cepok S, Rosche B, Grummel V, Vogel F, Zhou D, Sayn J, Sommer N, Hartung HP, Hemmer B: Short-lived plasma blasts are the main $B$ cell effector subset during the course of multiple sclerosis. Brain 2005, I 28: 1667-1676.

7I. Sundaram R, Beebe M, Kaumaya PT: Structural and immunogenicity analysis of chimeric B-cell epitope constructs derived from the gp46 and gp2I subunits of the envelope glycoproteins of HTLV-I. J Pept Res 2004, 63: I32-I 40.

72. Burbelo PD, Meoli E, Leahy HP, Graham J, Yao K, Oh U, Janik JE, Mahieux R, Kashanchi F, ladarola MJ, Jacobson S: Anti-HTLV antibody profiling reveals an antibody signature for HTLV-Iassociated myelopathy/tropical spastic paraparesis (HAM/ TSP). Retrovirology 2008, 5:96

73. Moles JP, Tesniere A, Guilhou J]: A new endogenous retroviral sequence is expressed in skin of patients with psoriasis. $\mathrm{Br} J$ Dermatol 2005, 153:83-89.

74. Sekigawa I, Ogasawara $\mathrm{H}$, Kaneko $\mathrm{H}$, Hishikawa T, Hashimoto $\mathrm{H}$ : Retroviruses and autoimmunity. Intern Med 200I, 40:80-86.

75. Ogasawara H, Naito T, Kaneko H, Hishikawa T, Sekigawa I, Hashimoto H, Kaneko Y, Yamamoto N, Maruyama N, Yamamoto N: Quantitative analyses of messenger RNA of human endogenous retrovirus in patients with systemic lupus erythematosus. J Rheumatol 200I, 28:533-538.

76. Lefranc D, Dubucquoi S, Almeras L, De Seze J, Tourvieille B, Dussart P, Aubert JP, Vermersch P, Prin L: Molecular Analysis of Endogenous Retrovirus HRES-I: Identification of Frameshift Mutations in Region Encoding Putative 28-kDa Autoantigen. Biochemical and Biophysical Research Communications 2001, 283:437-444.
77. Perl A, Nagy G, Koncz A, Gergely P, Fernandez D, Doherty E, Telarico $T$, Bonilla $E$, Phillips PE: Molecular mimicry and immunomodulation by the HRES-I endogenous retrovirus in SLE. Autoimmunity 2008, 4I:287-297.

78. Collins JR: Seizures and other neurologic manifestations of allergy. Vet Clin North Am Small Anim Pract 1994, 24:735-748.

79. Vezzani A: Inflammation and epilepsy. Epilepsy Curr 2005, 5: I-6.

80. Bauer J, Bien CG: Encephalitis and epilepsy. Semin Immunopathol 2009 in press.

8I. Fotheringham J, Donati D, Akhyani N, Fogdell-Hahn A, Vortmeyer A, Heiss JD, Williams E, Weinstein S, Bruce DA, Gaillard WD, Sato S, Theodore WH, Jacobson S: Association of human herpesvirus6B with mesial temporal lobe epilepsy. PLoS Med 2007, 4:e I80.

82. Poser CM, Paty DW, Scheinberg L, McDonald WI, Davis FA, Ebers GC, Johnson KP, Sibley WA, Silberberg DH, Tourtellotte WW: New diagnostic criteria for multiple sclerosis: guidelines for research protocols. Ann Neurol 1983, 13:227-23I.

83. Munch M, Moller-Larsen A, Christensen T, Morling N, Hansen HI, Haahr S: B-lymphoblastoid cell lines from multiple sclerosis patients and a healthy control producing a putative new human retrovirus and Epstein-Barr virus. Mult Scler 1995, I:78-8I.

84. Christensen $T$, Jensen AW, Munch M, Haahr S, Sorensen PD, Riemann H, Hansen HJ, Moller-Larsen A: Characterization of retroviruses from patients with multiple sclerosis. Acta Neurol Scand Suppl 1997, 169:49-58.

85. Christensen T, Tonjes RR, Megede JZ, Boller K, Moller-Larsen A: Reverse Transcriptase Activity and Particle Production in B Lymphoblastoid Cell Lines Established from Lymphocytes of Patients with Multiple Sclerosis. AIDS Research and Human Retroviruses 1999, 15:285-29I.

86. Moller-Larsen A, Christensen T: Isolation of a retrovirus from multiple sclerosis patients in self-generated lodixanol gradients. J Virol Methods 1998, 73:15I-161.

Publish with Biomed Central and every scientist can read your work free of charge

"BioMed Central will be the most significant development for disseminating the results of biomedical research in our lifetime. "

Sir Paul Nurse, Cancer Research UK

Your research papers will be:

- available free of charge to the entire biomedical community

- peer reviewed and published immediately upon acceptance

- cited in PubMed and archived on PubMed Central

- yours - you keep the copyright
BioMedcentral 